\title{
Blockchain und Co. - Was bringt uns die Entwicklung der Technik?
}

Blockchain, OnChain-Shop, Crypto stamps, CryptoKittie, Cryptowährung, Artifical Intelligence (Al) oder Künstliche Intelligence (KI), etc. - wer ist in seiner Datenschutzlaufbahn nicht schon mindestens einmal über einen dieser Begriffe gestolpert? Vor allem die Bezeichnung „Blockchain“ findet sich in letzter Zeit nicht nur zunehmend in Aufsatztiteln wieder, sondern scheint überdies das Schlagwort bzw. der Teilnahmemagnet in Konferenzankündigungen und sogar Schulungsangeboten zu sein. Es drängt sich demzufolge nicht ganz ohne Grund förmlich der Eindruck auf: Daran kommt niemand mehr vorbei.

Wer dabei das dringende Gefühl verspürt, sich erst einmal mit einem Wörterbuch oder Lexikon - bzw. eines seiner elektronischen Pedanten - zurückziehen zu müssen, ist sicherlich nicht vollständig allein. Tatsache ist jedoch, wer sich mit Themen des Datenschutzes oder/und der Datensicherheit beschäftigt, hat keine Möglichkeit sich der technischen Entwicklung zu entziehen. Aber, es sei allen gesagt, es ist kein Hexenwerk, auch diese "neuen Ansätze“ funktionieren in einer Welt der Datenverarbeitung nicht magisch, sondern sind Teil eines Systems, durch welches Datenverarbeitung betrieben wird. Und damit befinden sich doch zumindest die Datenschützerinnen und Datenschützer unter uns wieder auf der sicheren Seite, dem Schutz betroffener Personen vor unzulässiger Datenverarbeitung!

Aber was verbirgt sich nun eigentlich hinter Bezeichnungen wie „Blockchain“",Digitale Kopie" etc. und was macht es juristisch so spannend, dass selbst nicht technikbegeisterte Menschen auf einmal beginnen, sich mit den damit verbundenen Themen auseinanderzusetzen, sogar gespannt im Internet deren Entwicklung verfolgen. Dies hängt wohl zum einen mit den vielfältigen Nutzungsmöglichkeiten zusammen. Zum anderen ergeben sich zahlreiche rechtliche und informationstechnische Fragen, die geklärt werden wollen, um nicht zuletzt auch einen datenschutzkonformen Einsatz gewährleisten zu können.

Einige der wichtigsten Fragen und Diskussionen sollen im Rahmen dieses Schwerpunktheftes aufgegriffen werden. So wird sich zunächst der Beitrag „Blockchain-Shops im Web3" von Sven Venzke-Caprarese mit dem sehr aktuellen Thema des Angebots von Crypto stamps beschäftigen. Am Beispiel des OnChain-Shops der Österreichischen Post bietet er eine datenschutzrechtliche Beurteilung dieser neuartigen Briefmarken. Christopher Stradomsky geht sodann der Frage nach, inwieweit mit TR-ESOR ein datenschutzkonformer Einsatz einer Blockchain gewährleistet werden kann. Im Anschluss setzt sich der Beitrag von Prof. Dr. Christian Koenig mit der interessanten Frage des Postgeheimnisses im Zusammenhang von Postsendungen auseinander, die als Digitale Kopie erstellt werden. Im Anschluss an diesen Beitrag wird von Sebastian Schwäbe ein Überblick über die datenschutzkonforme technische Umsetzung von Webtracking geboten, während zuletzt durch Conrad S. Conrad noch der Frage näher nachgegangen wird, wie zu Zeiten der Vielzahl an neuen technischen Einsatzformen eine datenschutzrechtliche Zuordnung der Verantwortlichkeit überhaupt noch gewährleistet werden kann.

Insgesamt soll mit diesen ausgewählten Fragestellungen nicht nur eine eigene Beurteilung der Risiken und Chancen dieser technischen Entwicklung geboten werden, sondern insbesondere wieder einmal zur Diskussion angeregt werden.

Britta Alexandra Mester 